\title{
ТЕОРИЯ РАЗВИТИЯ МАЛЫХ И СРЕДНИХ ПРЕДПРИЯТИЙ НА ОСНОВЕ СОЗДАНИЯ БЛАГОПРИЯТНОЙ ПРЕДПРИНИМАТЕЛЬСКОЙ СРЕДЫ
}

\author{
С. И. Баженов, А. М. Платонов \\ Уральский федеральный университет имени первого Президента России Б. Н. Ельцина, г. Екатеринбург, \\ Российская Федерация
}

\author{
Информация о статье \\ Дата поступления \\ 8 февраля 2018 г. \\ Дата принятия к печати \\ 21 мая 2018 г. \\ Дата онлайн-размещения \\ 8 июня 2018 г.

\section{Ключевые слова} \\ Предпринимательская среда; \\ функциональные потребности; \\ малое предпринимательство; \\ среднее предпринимательство; \\ инновационная деятельность; \\ государственное \\ регулирование; фактор \\ мотивации
}

\begin{abstract}
Аннотация
Рассмотрены условия развития малого и среднего предпринимательства (МСП), повышения его роли в национальной экономике. Подтверждена гипотеза, согласно которой формирование благоприятной деловой среды может оказать большее влияние на повышение предпринимательской активности, чем объемы государственной поддержки. Функционирование благоприятной предпринимательской среды должно обеспечить: совершенствование механизмов взаимодействия государственных и муниципальных органов управления и субъектов предпринимательства на основе соблюдения взаимных интересов; создание условий для осуществления предпринимательской деятельности и повышения конкурентных преимуществ ее субъектов; формирование мотивации субъектов МСП к самоорганизационному и саморегулируемому развитию. Сформулированы выводы. Предложено изменить подходы к организации государственной и муниципальной поддержки малого и среднего предпринимательства, суть которых заключается в том, чтобы формирование благоприятной деловой среды для субъектов предпринимательства осуществлялось в различной сфере их деятельности с учетом их функциональных потребностей. Определены параметры результативности адресных мер государственной поддержки.
\end{abstract}

\section{THE THEORY OF SMALL AND MEDIUM BUSINESS DEVELOPMENT IN TERMS OF CREATION OF FAVORABLE BUSINESS ENVIRONMENT}

\author{
Sergey I. Bazhenov, Anatoliy M. Platonov \\ B. N. Yeltsin Ural Federal University, Yekaterinburg, the Russian Federation
}

\section{Article info}

Received

February 8, 2018

Accepted

May 21, 2018

Available online

June 8, 2018

\section{Keywords}

Business environment; functional needs; small business; medium business; innovation activity;

\begin{abstract}
Conditions of small and medium business development (SMB) and its increasing role in national economics are reviewed in this article. The authors proved the hypothesis that the formation of a favorable business environment can have a greater impact on increasing entrepreneurial activity than the amount of state support. Functioning of favorable business environment can be provided by: improvement of cooperation mechanisms of state and municipal government agencies and business entities in terms of maintaining mutual interests; setting the stage for business activity and increasing competitive positions of SMB entities; building the motivation of SMB entities towards self-organized and self-regulatory development. The authors made up conclusions and proposed to change the organizational
\end{abstract}


government control; motivating factor methods of state and municipal support for SMB in the terms of creating the favorable environment for business subjects in various areas considering their functional needs. They also identified the parameters of effectiveness of targeted measures of state support.
Введение. Одна из задач, поставленных президентом России В. В. Путиным перед Правительством РФ, - увеличить долю малого и среднего предпринимательства (МСП) в ВВП к 2020 г. до 60-70\%.

Сегодня в нашей стране действуют приблизительно 5,6 млн субъектов малого и среднего предпринимательства, которые обеспечивают занятость $25 \%$ населения и создают около 20 \% ВВП страны'. По мнению многих экспертов, возрождение России невозможно без соответствующего развития данного сектора экономики [1-3]. Именно с ним связаны инновационные процессы в экономике, способные обеспечить рост ряда макроэкономических и социальных показателей.

Теоретические разработки в рамках этого направления широко представлены в экономической литературе, в том числе в трудах А. С. Беджиева, В. В. Алещенко, А. В. Нурмухаметова и др.

Между тем чем выше научно-исследовательская активность в поисках новой парадигмы экономического роста МСП, тем выше вероятность достижения стратегических целей, что и обусловливает актуальность данного исследования.

Цель статьи заключается в том, чтобы на основе теоретического анализа, наблюдений за субъектом предпринимательства и знаний о его деятельности выявить его мотивационные факторы к саморазвитию.

Расширено понятие благоприятной предпринимательской среды, стимулирующей повышение конкурентных преимуществ МСП, выступающей в качестве фактора его мотивации (мотивационной среды) к самоорганизационному и саморегулируемому развитию, предусматривающей организацию государственной поддержки на основе дифрференцированного подхода с учетом различных фрункциональных потребностей малого и среднего предпринимательства.

Роль малого и среднего предпринимательства в национальной экономике. Считается, что лучшим хозяйственным порядком для экономического развития, обеспечива-

${ }^{1}$ В ближайшие годы число МСП в России увеличится до уровня стран с наиболее развитой экономикой // Министерство экономического развития Российской Федерации : офиц. сайт. URL: http://economy.gov. $\mathrm{ru} / \mathrm{minec} /$ about/structure/depMB/2015250906. ющего равенство возможностей экономического и социального для членов общества, в том числе за счет собственной предпринимательской деятельности, является такой порядок, где ведущая роль отводится МСП [4].

Необходимость наличия в любой национальной экономике высокоразвитого и эффективного малого предпринимательского сектора подтверждает и опыт ведущих стран современного мира. К примеру, доля малого и среднего предпринимательства в экономике развитых стран, таких как США, Япония, Великобритания, Германия, составляет от 50 до $57 \%$.

В развитых странах мира субъекты малого бизнеса уже давно зарекомендовали себя как необходимая и важная составляющая сильной национальной экономики [5].

Малые и средние предприятия в развитых странах составляют важнейший сектор национальных экономик. Если крупные предприятия определяют уровень научно-технического и производственного потенциала страны, то МСП, являясь наиболее массовой формой деловой жизни, обеспечивает социальноэкономическую стабильность развития [6].

Вполне обоснованными представляются утверждения экономистов о том, что малое и среднее предпринимательство должно стать одним из факторов обеспечения устойчивого развития российской экономики. Однако для выполнения этого условия мы считали бы необходимым добиться эфффективного государственного регулирования и стимулирования предпринимательских инициатив, что позволило бы использовать основные функции МСП и его определенные стратегические преимущества в системе рыночного хозяйствования, которая еще недостаточно адаптирована к рыночным отношениям.

Несмотря на возросшее внимание органов власти к проблемам малого и среднего предпринимательства в России, данный сектор экономики не оказывает того влияния на экономическую безопасность регионов страны, на которое можно было бы рассчитывать в силу его высокого потенциала в сорере применения инновационных технологий при решении сложных социально-экономических задач.

Национальный бизнес на рынке инноваций по-прежнему неконкурентоспособен, отраслевая диверсификация предприятий МСП 
достаточно низкая. Отраслевая структура показывает, что наибольший удельный вес сегодня имеют малые и средние предприятия, функционирующие в сфере торговли.

Проникновение МСП в реальный сектор экономики (кроме строительства) весьма незначительно. Доля малого и среднего предпринимательства в хозяйственном обороте промышленности составляет порядка 5-6\%. Инновационные малые предприятия реально заметны и более или менее продуктивны только в особых точках их сосредоточения (например, в рамках особой экономической зоны (ОЭЗ) технико-внедренческого типа), но в целом говорить об их весовом вкладе в инновационный «прорыв» в российской экономике было бы преждевременно [7, с. 46].

Трансформация функциональных потребностей малого и среднего предпринимательства в процессе развития. Многие исследователи оценивают низкую активность МСП в рыночной экономике как результат слабой государственной поддержки его развития.

У теории развития предпринимательства на основе формирования благоприятной предпринимательской среды немало сторонников. Возникает резонный вопрос, можно ли между этими двумя терминами поставить знак равенства, поскольку цель едина: и государственная поддержка МСП, и благоприятная предпринимательская среда должны обеспечить экономический рост малого и среднего предпринимательства.

В рамках традиционного подхода государственная поддержка МСП - это совокупность мер организационного, финансового, консультативного и институционального характера, направленная на его развитие. Справедливости ради следует отметить, что механизм предоставления государственной поддержки по такой схеме постоянно совершенствуется. На первый взгляд, реализация данного комплекса мер позволит субъекту предпринимательства успешно заполнить свободное пространство рынка и обеспечить устойчивое позитивное влияние на его развитие.

Однако практические наблюдения за данным экономическим субъектом и знания о его деятельности, полученные из разных источников, убеждают в том, что субъекты предпринимательства недостаточно мотивированы только экономическим интересом. Помимо прибыли, расширения производства и сорер деятельности важным для них является общественное признание. В этой связи усилилось их стремление к социальной креативности, участию в различных сферах общественной жизни.
Кроме того, президент России ставит перед правительством задачу перевести экономику с сырьевого на инновационный путь развития. По опыту зарубежных стран можно сказать, что эту нишу достаточно успешно заполняют малые предприятия. Российские эксперты также рассматривают малое наукоемкое предпринимательство как одно из ведущих направлений в развитии инновационной национальной экономики. Однако сегодня показатели занятых в инновационной сорере малых предприятий очень скромны и не превышают $6 \%$. Система государственной поддержки не выделяет эту категорию экономических агентов из общего перечня малых и средних предприятий, хотя она отличается по характеру выпускаемой продукции, организации деятельности, требованиям к персоналу, использованию рыночных механизмов, поэтому подходы к государственной поддержке таких предприятий должны быть иными, более «чувствительными» к их потребностям.

Потребности современных предпринимателей независимо от сфреры их деятельности трансформируются в связи с изменением социально-экономической реальности в стране, регионе, а также под воздействием растущих требований рынка к качеству услуг и выпускаемой продукции. В условиях усиления конкуренции, роста научно-технического прогресса участники рынка, каковыми являются и субъекты малого и среднего хозяйствования, тоже обязаны расти в профессиональном, интеллектуальном плане, заботиться о маркетинговом продвижении своей продукции.

Возникает необходимость обеспечить в результате развития достижение не только определенных экономических сдвигов, но и экономической стабильности, устойчивости. Ориентация на максимизацию прибыли объективно уже не может больше рассматриваться как основная движущая сила развития - развитие должно осуществляться с обязательным учетом кроме экономического еще и социального, и экологического фракторов. Это касается как развития общества в целом, так и развития экономики отдельных стран и экономики предприятий, фрормирующих потенциал развития экономики каждой страны [8].

Теоретические исследования в сорере МСП в регионе убедили в том, что предпринимательская активность не всегда зависит от объемов государственной поддержки. В большей степени она обусловлена наличием деловой среды, сорормированной под воздействием таких фракторов, как: 
1. Неформальный подход государственных и муниципальных органов управления к проблемам малого и среднего предпринимательства, проведение целенаправленной политики в сорере МСП, ориентированной на решение следующих задач:

- полноценная интеграция МСП в региональную и муниципальную экономику;

- создание эфффективного механизма снижения предпринимательских рисков;

- обеспечение самостоятельной предпринимательской деятельности на территории под управлением определенных стимулов и льгот;

- формирование поколения бизнес-лидеров, предпринимателей, новаторов на основе принятия пилотных программ обучения в школах.

2. Создание и стабильное функционирование институтов взаимодействия власти и бизнеса на основе сбалансированности интересов их участников.

3. Наличие региональных и муниципальных информационных каналов, доступных всем категориям предпринимателей, в том числе начинающим, и способствующих фрормированию положительного имиджа предпринимательства.

В современных условиях развития малого и среднего предпринимательства возрастает и роль общественных объединений (организаций, союзов, некоммерческих партнерств, ассоциаций и т. д.), которые берут на себя часть функций взаимодействия с органами власти, а именно: доводят до сведения местного регулятора консолидированное мнение их участников по определенным проблемам малого и среднего предпринимательства с предложением возможных решений; участвуют в обсуждении законопроектов, касающихся предпринимательской деятельности; входят в консультативные советы при органах власти.

Мировая практика свидетельствуето том, что бизнес-ассоциации являются эфффективной формой самоорганизации делового сообщества и могут с успехом представлять интересы субъектов крупного, среднего и малого предпринимательства, выступая посредниками в диалоге между бизнесом и властью. Это особенно актуально для стран с переходной экономикой, в которых традиции активного участия граждан в формировании цивилизованных рыночных отношений находятся в начальной стадии. В таких странах деятельность бизнес-ассоциаций представляет собой один из наиболее реальных способов влияния на принимаемые решения с целью создания благоприятных условий для развития бизнеса [9].

В основном успешность функционирования объединений предпринимателей определяется лидерскими качествами их руководителей, активностью их участников. Согласно теории лидерских качеств, лидерами не рождаются, лидерами становятся. Аналогично можно сказать и про статус предпринимателя. И хотя мы не исключаем наличие природного потенциала, наряду с ним важно, чтобы предприниматель владел лидерскими качествами, которые он при определенных условиях и сильной мотивации может приобрести в результате специальной подготовки.

Однако объединения предпринимателей в сравнении с опытом зарубежных стран достаточно новое явление в России. Основная проблема, с которой сталкиваются объединения, - недостаток финансирования. Поэтому наличие лидерских качеств у руководителя подобной структуры является важным, но не единственным условием эфрфективного взаимодействия власти и бизнеса. В качестве необходимого условия достижения общественно значимых целей в предпринимательской деятельности следует также рассматривать солидарную заинтересованность субъектов отношений - власти и бизнеса - в сотрудничестве, основанном на получении выгоды обеими сторонами.

Государственная и муниципальная власть в силу своих полномочий ответственна за социально-экономическое развитие территории. Ее выгода определяется в виде платежей в бюджеты этих уровней, снижения давления на рынок труда, удовлетворения спроса потребителей, повышения конкурентных качеств территории.

Объединения предпринимателей (так записано в уставах большинства из них) призваны защищать интересы добросовестных производителей, эффективно лоббировать различные предпринимательские идеи, добиваться решения тактических задач, а также выдвигать перспективные планы развития рынка, практически став его регулятором.

Современному бизнесу присуща ориентация не только на получение прибыли. С точки зрения управления бизнес - это система хозяйствования, основанная на инновационной деятельности, включающая элементы риска и характеризующаяся наличием процесса воспроизводства с целью получения предпринимательского дохода. Без взаимодействия власти и бизнеса, основанного на отношениях взаимной зависимости, реали- 
зация данных свойств бизнеса затруднена, так как необходимо создание благоприятных внешних условий. Такие условия может создать только государство [10].

Итак, можно утверждать, что современное малое и среднее предпринимательство стремится к различным типам объединений на платформе более профессиональной, чем, предположим, десятилетие тому назад, с поставленной четкой целью и задачами, что свидетельствует о его зрелости в качестве участника рынка и партнера во взаимоотношениях с органами власти.

Прежде всего эти объединения решают задачи обеспечения эфффективного взаимодействия внутри предпринимательских сообществ, ориентированы на выполнение совместных проектов и, следовательно, способны сорормировать цивилизованное отношение к ведению предпринимательства.

Очевидно, что сегодня предпринимательство может проявить свое умение к саморазвитию, для этого существуют все предпосылки. Однако исключать из совокупности мер, ориентированных на их развитие, государственную поддержку нет никаких оснований. Более того, на наш взгляд, целесообразно усилить поддержку, качественно изменив подходы, предусмотрев необходимость формирования институтов, стимулирующих самоорганизационные процессы в предпринимательской сорере.

Согласно институциональной теории Веблена, экономическое развитие в рыночной экономике представляет собой механизм естественного отбора, основанного на жесткой и бескомпромиссной борьбе хозяйствующих субъектов за выживание в условиях рынка [11].

Следуя этой теории, малые и средние предприятия должны обрести способность к выживанию. Для этого им необходимо обеспечить свою конкурентоспособность. Считаем, что основой решения данной задачи является применение новых форм и методов организации производства и управления им в рыночных условиях, требующих адекватной профессиональной подготовки предпринимателя в качестве менеджера, экономиста, администратора, а также наличие ресурсов функционирования и развития.

При таком подходе государство, если оно заинтересовано в сохранении предпринимательского сектора, еще более - в его развитии, обязано создать эффрективную систему его стимулирования и поддержки, поскольку субъекты малого и среднего предпринимательства, даже объединившись, не в состоянии противостоять всем рискам и угрозам, порождаемым новыми реалиями, к примеру таким, как кризис спроса на рынке товаров и услуг, недостаток финансовых ресурсов, кредитные и инвестиционные риски, слабые возможности проведения маркетинговых исследований рынка и т. д.

В то же время есть необходимость концептуально определиться, стоит ли оказывать государственную помощь каждому предпринимателю в борьбе за выживание в условиях жесткой конкуренции. Не секрет, что существует отдельная категория предпринимателей, которые неплохо устроились и ориентированы только на получение быстрой прибыли, так сказать, стремятся «сорвать куш».

Условия саморазвития малого и среднего предпринимательства. Предприниматель должен уметь руководить, организовывать, планировать, ставить цели, постоянно учиться новому, рисковать и не бояться перемен так позиционирует бизнес-сообщество образ предпринимателя. Можно смело утверждать, что обладающий этими качествами субъект предпринимательской деятельности выходит на рынок с серьезными намерениями и способен внести существенный вклад в процесс саморазвития МСП. Но ему необходима помощь в формировании и осуществлении предпринимательской компетенции, для чего нужна благоприятная среда, в которой действуют механизмы, снижающие предпринимательские риски, обеспечивающие финансовую устойчивость, государственные и муниципальные гарантии.

В экономической литературе представлены различные подходы к определению термина «благоприятная предпринимательская среда» $[12 ; 13]$. Однако основная сущность и содержание его сводятся к обеспечению хозяйствующим субъектам экономической свободы для занятия предпринимательской деятельностью. Но свободой надо уметь пользоваться, не нарушая баланса интересов. Только тогда можно добиться успехов.

В условиях незавершенности реформ в экономике и несовершенства правоприменительной системы, рыночных механизмов, в том числе слабой разработанности проблем конкуренции, мы считали бы необходимым расширить данное понятие, предусмотрев формирование среды как способ влияния на повышение конкурентных преимуществ малых и средних предприятий посредством обеспечения достаточного объема ресурсов и равного доступа к ним, принятия гарантий минимизации рисков, повышения 
эфрфективности административно-правового механизма, содействия повышению профессионального правосознания субъекта предпринимательства, развития взаимовыгодного сотрудничества органов управления с предпринимательскими объединениями.

Принцип создания благоприятной среды для развития МСП должен лежать в основе государственной политики и реализовываться по следующим направлениям:

1. Создание системы государственной поддержки стартующих предпринимателей с учетом предоставления им равных возможностей. В основе этой системы, содержащей дополнительные механизмы поощрения и стимулирования успешных субъектов предпринимательской деятельности, лежат традиционные подходы.

2. Формирование институтов и механизмов поддержки, функционирование которых должно быть направлено на снижение рисков и повышение конкурентоспособности действующих субъектов МСП. Развитие взаимодействия с предпринимательскими объединениями на основе взаимовыгодных интересов, вовлечение их в нормотворческий процесс, разработка региональных и муниципальных программ развития. Укрепление предпринимательского сектора экономики и повышение его авторитета путем включения в перечень приоритетных задач стратегического плана развития территории, увеличения объема государственных и муниципальных заказов.

3. Обеспечение условий для развития малого наукоемкого предпринимательства: предоставление специальных помещений для организации научной предпринимательской деятельности; оказание помощи в их технологическом оснащении, модернизации производственной базы; создание инновационной инфраструктуры финансовой, интеллектуальной и организационной помощи. Вовлечение МСП в техническое перевооружение территорий, обучение технологическому предпринимательству. Содействие деятельности малого наукоемкого предпринимательства посредством размещения государственного заказа на разработку инновационных научно-технических проектов, повышение доли государственного инвестирования в производство новых технологий. Принятие мер стимулирования кооперации с крупными предприятиями в области применения сервисных и высокотехнологичных услуг.

4. Содействие созданию и развитию институтов финансово-кредитной, инвестиционной и налоговой поддержки внешнеэко- номической деятельности инновационного и экспортно ориентированного малого и среднего предпринимательства, продвижению его на внешние рынки.

Степень участия и заинтересованности органов власти в развитии МСП должна оцениваться на всех уровнях власти и по каждому направлению предпринимательской деятельности в рамках создания благоприятной предпринимательской среды.

К основным параметрам, которые характеризовали бы результативность системы государственного стимулирования предпринимательского сектора за фиксированный период, на наш взгляд, следует отнести следующие.

По первому направлению:

- количество созданных малых и средних предприятий;

- динамика процентного соотношения количества созданных хозяйствующих субъектов к прекратившим свою деятельность;

- показатели изменения бюджетных поступлений, занятости и самозанятости.

По второму направлению:

- оценка годового оборота малых и средних предприятий;

- степень вовлечения в производство национального продукта;

- динамика количества малых и средних предприятий инновационного направления, способных к самообеспечению.

По третьему направлению:

- рост количества малых предприятий, занятых в инновационной сорере;

- степень влияния наукоемких малых и средних предприятий на развитие промышленного, социального секторов экономики, например машиностроение, станкостроение, электроника, строительство объектов в социально-культурной сфере;

- практика внедрения проектных работ;

- количество выданных патентов и инноваций.

По четвертому направлению:

- рост количества экспортно ориентированных малых и средних предприятий;

- динамика показателей экспорта МСП;

- расширение товарной номенклатуры внешнеэкономической деятельности МСП.

Предпринимательская среда представляет собой сложную интегрированную систему. Она подразделяется на внешнюю среду и внутреннюю. Поэтому наряду с внешним воздействием (в полной мере зависящим от социально-экономического положения страны и уровня государственного регулирования) присутствует и фактор зависимости 
от самого субъекта предпринимательской деятельности, его профессионализма, компетентности, способности стратегически мыслить и эфффективно управлять.

Согласно концепции В. Д. Шадрикова, внутренний мир человека, как самоорганизующаяся система, стремится к устойчивому состоянию [14]. Если возникают новые задачи, которые невозможно решить на основе сложившихся форм поведения (стереотипов), то для восстановления устойчивости система обращается к новациям и творчеству.

Поэтому мы убеждены, что благоприятная для развития предпринимательства в условиях жесткой конкуренции на рынке среда, созданная при участии органов государственного и муниципального управления, явится тем мотивационным фактором, который сможет побудить экономических субъектов к повышению уровня менеджмента, формированию предпринимательских инициатив на более качественном уровне, поскольку наряду с созданием предпринимательской среды будут заданы иные целевые ориентиры, которые потребуют новых подходов к решению поставленных задач.

Заключение. Сорера деятельности малого и среднего предпринимательства достаточно обширна и, несмотря ни низкие темпы его развития, продолжает расширяться. Оно укрепляет свои позиции на рынке, для обеспечения своей конкурентоспособности прибегает к использованию инновационных технологий, участвует в социальных инвестиционных проектах. Однако не секрет, что для формирования устойчивой тенденции его экономического роста прежде всего необходимо укрепить внешнюю среду, которая в силу объективных причин и несовершенства регулирующих механизмов остается подвижной и полной неопределенности, что сильно затрудняет его функционирование.

Согласно теории рыночной экономики, в сорере хозяйственной деятельности предпринимательство имеет бо́льшую эффективность, чем государственное управление. В то же время практика показывает, что данное положение справедливо в условиях наличия развитых рыночных механизмов и механизмов законодательного регулирования.

Без изменения институтов, обеспечивающих эффрективный кредитный механизм, подготовку специалистов нужной квалификации, надежную правовую защиту предпринимательства, облегчающих его доступ к источникам экономической и правовой информации, невозможно развитие малого и среднего бизнеса, причем не только инновационно активного, а бизнеса вообще [15].

На наш взгляд, до настоящего времени государство недостаточно вкладывалось в развитие малых и средних предприятий. Дальнейшее стимулирование их развития потребует значительных усилий и ресурсных затрат со стороны органов управления. Очевидно, что чем организованнее и профессиональнее становится МСП, тем выше уровень задач, за решение которых оно берется. Национальная экономика от этого может только выиграть. Однако система государственной поддержки при этом тоже должна совершенствоваться и соответствовать новым реалиям, предусмотрев не только ресурсное обеспечение, недостаток которого отмечался на заре становления малого и среднего предпринимательства и продолжает сохраняться в настоящее время, но и дифрференцированный подход с учетом различных функциональных потребностей субъектов предпринимательской деятельности, акцентируя при этом внимание на формировании благоприятной предпринимательской среды для каждой категории экономических субъектов: стартующее; действующее; наукоемкое; осуществляющее внешнеэкономическую деятельность; инновационное и экспортно ориентированное МСП.

Благоприятную предпринимательскую среду следует рассматривать в качестве фактора, мотивирующего субъектов предпринимательской деятельности к самоорганизационному и саморегулируемому развитию, способствующего повышению их конкурентных преимуществ посредством обеспечения достаточного объема ресурсов и равного доступа к ним, государственных гарантий снижения рисков; совершенствования механизма административно-правового регулирования; содействия повышению уровня профессионального правосознания; развития взаимовыгодного сотрудничества органов управления с предпринимательским сообществом.

Основополагающий принцип, который должен лежать в основе создания благоприятной предпринимательской среды, именно в том понимании, в каком данный термин представлен в экономической литературе, - обеспечение экономической свободы, позволяющей субъектам хозяйствования добиваться поставленной цели, в том числе и в части получения дохода. 


\section{СПИСОК ИСПОЛЬЗОВАННОЙ ЛИТЕРАТУРЫ}

1. Конова О. Ю. Самоорганизация малого предпринимательства в России [Электронный ресурс] / О. Ю. Конова, Е.А.Резникова / / Проблемы региональной экономики. - 2016. - Вып. 35. - Режим доступа: http: / / www.lerc.ru/?part=bulletin\&art=35\&page=6.

2. Нурмухаметов А. В. Значение малого и среднего бизнеса в экономике страны / А. В. Нурмухаметов // Актуальные вопросы экономических наук : материалы 3-й Междунар. науч. конф. (Уфа, июнь 2014 г.). Уфра : Лето, 2014. - С. 16-19.

3. Лясников Н. В. Современное российское предпринимательство. Роль малого и среднего предпринимательства в экономике страны / Н. В. Лясников, М.Н.Дудин / / Креативная экономика. — 2008. — № 5 (17). C. 83-90.

4. Совершенствование механизма государственной поддержки субъектов малого и среднего предпринимательства / под общ. ред. В. В. Алещенко, В. В. Карпова. - Омск : ОМ. науч. вестник, 2015. - 188 с.

5. Гинзбург Я. Б. Закономерности развития малого бизнеса в регионе : автореф. дис. ... канд. экон. наук : 08.00.05 / Я. Б. Гинзбург. - Иркутск, 2005. - 19 с.

6. Петров Е. В. Развитие форм государственной поддержки инвестиционной деятельности малого предпринимательства / Е. В. Петров, А. Е. Шульгина / / Экономика, управление, финансы : материалы 5-й Междунар. науч. конф. (Краснодар, август 2015 г.). - Краснодар : Новация, 2015. - С. 120-124.

7. Бухвальд Е. М. Модернизация экономики и новые подходы к политике поддержки малого и среднего предпринимательства / Е. М. Бухвальд / / Общество: политика, экономика, право. — 2014. — № 1. — С. 45-52.

8. Коряков А. Г. Экономическая устойчивость развития предприятий: классификация и ключевые факторы / А. Г. Коряков / / Современная наука: актуальные проблемы теории и практики. Сер.: Экономика и право. -2012 . - № 3. - С. 8-12.

9. Биджиев А. С. Взаимодействие органов власти и бизнеса как фактор управления социально-экономическим развитием региона / А. С. Биджиев, Г. М. Шамарова / / Вопросы управления. - 2012. — № 1 (18). С. $132-141$.

10. Айрапетян Л. Н. Взаимодействие власти и бизнеса при разработке и реализации целевых программ : дис. ... канд. экон. наук : 08.00.05 / Л. Н. Айрапетян. - М., 2014. - 189 с.

11. Калужский М. Л. Институционализм и экзогенная интерпретация экономических процессов / М. Л. Калужский / / Омский научный вестник. - 2013. - № 1 (115). - С. 52-55.

12. Ворожбит О. Ю. Структура предпринимательской среды: определяющие фракторы / О. Ю. Ворожбит, Н. В. Зубова, А. В. Корень / / Вестник Тихоокеанского государственного университета. - 2010. — № 4 (19). - С. 121-128.

13. Закономерности и тенденции развития современного предпринимательства / под ред. А. Н. Асаула. - СПб., 2008. - 280 с.

14. Шадриков В. Д. От индивида к индивидуальности / В. Д. Шадриков. - М. : Ин-т психологии РАН, 2009. -240 с.

15. Антончиков С. Н. Инновационная активность МСП в России и Евросоюзе и факторы ее развития [Электронный ресурс] / С. Н. Антончиков // Группа компаний НИСИПП. - Режим доступа: http://nisse.ru/ articles/details.php?ELEMENT_ID $=118713$.

\section{REFERENCES}

1. Konova O. Yu., Reznikova E. A. Self Organization of Small Business in Russia. Problemy regional'noi ekonomiki= Roadblocks of Regional Economics, 2016, vol. 35. Available at: http://www.lerc.ru/?part=bulletin\&art=35\&page=6. (In Russian).

2. Nurmukhametov A. V. The Role of Small and Medium Business in the National Economy. Aktual'nye voprosy ekonomicheskikh nauk. Materialy 3-i Mezhdunarodnoi nauchnoi konferentsii, Ufa, iyun 2014 g. [Key Considerations of Economics. Material of the $3^{\text {rd }}$ International Scientific Meeting, Ufa, June 2014]. Ufa, Leto Publ., 2014, pp. 16-19. (In Russian).

3. Lyasnikov N. V., Dudin M. N. Contemporary Russian Business. The Role of Small and Medium Business in the National Economy. Kreativnaya ekonomika = Creative Economy, 2008, no. 5 (17), pp. 83-90. (In Russian).

4. Aleshchenko V. V., Karpov V. V. (eds.). Sovershenstvovanie mekhanizma gosudarstvennoi podderzhki sub"ektov malogo i srednego predprinimatel'stva [Improvement of the State Support Mechanism for the Small and Medium Business Entities]. Omsk, Omskii nauchnyi vestnik Publ., 2015. 188 p.

5. Ginzburg Ya. B. Zakonomernosti razvitiya malogo biznesa v regione. Aftoref. Kand. Diss. [Conformities of Development of the Small Business in the Regional Area. Cand. Diss. Thesis]. Irkutsk, 2005. $19 \mathrm{p}$.

6. Petrov E. V., Shul'gina A. E. Development of State Support Patterns for Small Business Investment Activity. Ekonomika, upravlenie, finansy. Materialy 5-i Mezhdunarodnoi nauchnoi konferentsii, Krasnodar, avgust 2015 g. [Economics, Management, Finances. Material of the $5^{\text {th }}$ International Scientific Meeting, Krasnodar, August 2015]. Krasnodar, Novatsiya Publ., 2015, pp. 120-124. (In Russian).

7. Bukhvald E. M. Economy Modernization and New Approaches to Small and Medium Businesses Support. Obshchestvo: politika, ekonomika, pravo = Society: Politics, Economics, Law, 2014, no. 1, pp. 45-52. (In Russian).

8. Koriakov A. G. Economic Sustainable Development for Entrprises: Clasification and Main Factors. Sovremennaya nauka: aktual' nye problemy teorii i praktiki. Seriya: Ekonomika i pravo = Modern Science: actual problems of theory and practice. Series: Law and Economics, 2012, no. 3, pp. 8-12. (In Russian).

9. Bidjiev A. S., Shamarova G. M. Interaction between Government and Business as a Factor of Socio-Economic Development of the Region. Voprosy upravleniya = Management Issues, 2012, no. 1 (18), pp. 132-141. (In Russian). 
10. Airapetyan L. N. Vzaimodeistvie vlasti i biznesa pri razrabotke i realizatsii tselevykh programm. Kand. Diss. [Governance and Business Cooperation During the Design and Implementation of Target Programmes. Cand. Diss.]. Moscow, 2014. 189 p.

11. Kaluzhsky M. L. Institutionalism and Exogenous Interpretation of Economic Processes. Omskii nauchnyi vestnik = Omsk Scientific Bulletin, 2013, no. 1 (115), pp. 52-55. (In Russian).

12. Vorozhbit O. Yu., Zubova N. V., Koren A. V. Structure of Entrepreneurial Environment: Essential Factors. Vestnik Tikhookeanskogo gosudarstvennogo universiteta = Pacific State University Bulletin, 2010, no. 4 (19), pp. 121-128. (In Russian).

13. Asaul A. N. (ed.). Zakonomernosti i tendentsii razvitiya sovremennogo predprinimatel'stva [Conformities and Trends of Contemporary Business Development]. Saint Petersburg, 2008. 280 p.

14. Shadrikov V. D. Ot individa $k$ individual' nosti [From Individuum to Individuality]. Moscow, RAS Institute of Psychology Publ., 2009. 240 p.

15. Antonchikov S. N. Innovative Activity of SMB in Russia and its Development Factors. Gruppa kompanii NISIPP $=$ NISSEP Corporate Group. Available at: http://nisse.ru/articles/details.php?ELEMENT_ID=118713. (In Russian).

\section{Информация об авторах}

Баженов Сергей Иванович - доктор экономических наук, профрессор, кафедра экономики и управления строительством и рынком недвижимости, Уральский федеральный университет имени первого Президента России Б. Н. Ельцина, 620002, г. Екатеринбург, ул. Мира, 19, e-mail: naukaservis@ rambler.ru.

Платонов Анатолий Михайлович - доктор экономических наук, профессор, профессор-консультант, кафедра экономики и управления строительством и рынком недвижимости, Уральский федеральный университет имени первого Президента России Б. Н. Ельцина, 620002, г. Екатеринбург, ул. Мира, 19, e-mail: eusrn@mail.ru.

\section{Для цитирования}

Баженов С. И. Теория развития малых и средних предприятий на основе создания благоприятной предпринимательской среды / С. И. Баженов, А. М. Платонов // Известия Байкальского государственного университета. - 2018. - Т. 28 № 2. - C. 240-248. - DOI: $10.17150 / 2500-$ 2759.2018.28(2).240-248.

\section{Authors}

Sergey I. Bazhenov - D.Sc. in Economics, Professor, Department of Economic and Building Management and Estate Market, B. N. Yeltsin Ural Federal University, 19 Mira St. , 620002, Yekaterinburg, the Russian Federation, e-mail: naukaservis@rambler.ru.

Anatoliy M. Platonov - D.Sc. in Economics, Professor, Department of Economic and Building Management and Estate Market, B. N. Yeltsin Ural Federal University, 19 Mira St., 620002, Yekaterinburg, the Russian Federation, e-mail: eusrn@mail.ru.

\section{For citation}

Bazhenov S. I., Platonov A. M. The Theory of Small and Medium Business Development in Terms of Creation of Favorable Business Environment. Izvestiya Baykal' skogo gosudarstvennogo universiteta $=$ Bulletin of Baikal State University, 2018, vol. 28, no. 2, pp. 240248. DOI: $10.17150 / 2500-2759.2018 .28(2) .240-248$. (In Russian). 